\title{
PASSENGER BEHAVIOUR SIMULATION INSIDE TRAIN CAR COMBINED WITH TRAIN TRAFFIC SIMULATION
}

\author{
TAKETOSHI KUNIMATSU ${ }^{1}$, HIRONORI MORIYA ${ }^{2}$, HIROAKI KUSAKABE $^{2} \&$ RYOHEI YAMADA ${ }^{2}$ \\ ${ }^{1}$ Railway Technical Research Institute, Japan \\ ${ }^{2}$ JR Souken Information Systems Co., Ltd., Japan
}

\begin{abstract}
The aim of this research is to improve functions of train traffic simulation to evaluate train timetables. We want to improve reproducibility of train delay caused by passengers' boarding and alighting. We also want to estimate boarding passengers' discomforts caused by congestion or collision with other passengers inside the train car. In railway commuter lines in a big city like Tokyo, train delay due to congestion is one of the biggest problems. When the railway company makes a timetable, although it is important to set enough dwell time at stations to prevent train delay, there is no way to confirm the appropriateness of timetable alternatives in advance. In our previous researches, we have developed a train operation and passenger flow simulator, which can reproduce train congestion, train delay, and their interactions, like the "snowball effect". However, the reproducibility of train delay in the simulator is not enough, because dwell time necessary for boarding and alighting is different, along with position of each passenger inside train car. In this research, we proposed a simulation method of passengers' movements to estimate the time necessary for boarding and alighting. The method can consider each passenger's shortest walking path towards the train door and their target position inside the train car, as well as passengers who temporarily alight the train, in which they disturb other alighting passengers. The method also realizes short calculation time enough for evaluating a whole timetable composed of a combination of many trains, stations, and doors. We conducted test calculations for the different movements of passengers. We compared the estimated time for boarding and alighting by the simulator, with the actual dwell time and estimated time by existing method by using a regression formula. As a result, we conclude that the proposed method can estimate each passenger's behaviour in detail without decreasing reproducibility of train delay.

Keywords: train traffic simulation, passenger's behaviour, delay, dwell time, shortest path search.
\end{abstract}

\section{INTRODUCTION}

In commuter lines in Tokyo, trains are often delayed due to extension of dwell time caused by congestion [1]. One of the countermeasures for this problem is appropriate timetabling reflecting passenger flow. That is, setting enough dwell time supplements at stations considering number of passengers alighting and boarding, and setting enough buffer time not to propagate train delay from a certain train to the succeeding train.

The authors have developed "Train Operation and Passenger Flow Simulator", which validates a train timetable before it goes into operation [2]. The simulator estimates train congestion and delay by using passenger data collected by automatic ticket gates at stations. Estimation of train delay is conducted by using the number of passengers alighting and boarding at each station, on each train, and from each door. Then the necessary time for alighting and boarding is predicted. However, the necessary time for alighting and boarding is determined not only by the number of passengers, but also the detailed position of passengers, layout inside train car, or the way to line up for the train on a platform. Actually, railway companies take efforts for decreasing the necessary time, and train delay. Moreover, if it is very congested in train car, some passengers near the door may temporarily get out the train to make way for alighting passengers. By using the conventional simulator, we cannot consider such situations or behaviour. So, we cannot estimate effects of reduction of train delay by taking such countermeasures as smooth boarding and alighting. 
In this research, we developed simulation function for passengers' boarding and alighting at stations. It estimates necessary time for boarding and alighting in great detail by simulating each passenger's behaviour at every second. It can also combine with train operation and passenger flow simulator, which enable us to predict effects for reducing train delay by taking a countermeasure for smooth boarding and alighting. At first, we conducted questioner survey about passengers' preference of position inside train car. Then, we developed simulation function for boarding and alighting. Then, we combined the simulation function and passenger's choice model of vehicle into the simulator.

To validate these methods, we compared the estimated dwell time by the developed simulator with the actual ones. We confirmed that accuracy of the devised method is not worse than conventional method. Moreover, we evaluated and compared two plans for smooth boarding and alighting by test calculations for train delay and passengers' comfort. Finally, we concluded that the proposed method is useful enough to evaluate such plans quantitatively.

\section{AIM AND APPROACH OF THE RESEARCH}

\subsection{Problems of rail transportation during peak hours in commuter lines in Japan}

In the morning peak hours, as many passengers commute, trains and stations become very congested. Trains tend to be delayed, because the time necessary for boarding and alighting become longer than the planned dwell time at a station. In addition, once a train becomes delayed, train interval between the preceding train and that train become larger, and this in turn increases the number of passengers waiting for that train at the next station. So, the extent of delay for that train at the next station tends to increase as a consequence of longer necessary time for boarding and alighting. This phenomenon is called "snowball effect" of train delay and train congestion. As snowball effect decreases passengers' comfort and punctuality of train operation, railway companies take efforts for avoiding that, by setting as many trains as possible to decrease congestion, or making a robust timetable by including much running time / dwell time supplements and buffer time between trains.

However, if train timetable is changed, passengers take different train paths towards their destination. This in turn affects train congestion of trains and stations, and the necessary time for boarding and alighting. So, it is difficult for us to predict such changes in advance. Thus, there is a problem for railway companies that they have no way for validating robustness of a new timetable, or effectiveness of some counterplans for congestion and delay.

\subsection{Related work}

There are many related works concerning train delay and congestion. As a previous work for estimating train delay and congestion by simulation is "Train Operation and Passenger Flow Simulator", developed by the authors [2]. This simulator uses passenger data collected by automatic ticket gates at stations. The simulator estimates each passenger's train paths towards his/her destination along with a train timetable (Fig. 1). It then estimates train congestion by aggregating results of all passengers' estimated train paths. It also predicts train delay caused by congestion. That is, it calculates necessary dwell time at a station by using estimated number of passengers boarding and alighting.

When it calculates necessary dwell time, the regression formula introduced by another previous work about passenger flow is used [3]. However, this method has a drawback. That is, although the time for boarding and alighting is different along with the condition inside 
train car, like position of alighting passengers, it is not considered. In fact, some passengers may temporarily get out of the train, because they disturb other alighting passengers.

On the other hand, there is another work for simulating passengers' behaviour of boarding and alighting [4]. This work treats a passenger as an agent. It estimates necessary time for boarding and alighting by agent-based simulation technique. However, this work does not consider existence of other passengers on each passenger's walking path. That is, if there are some passengers on the shortest walking path of an alighting passenger towards the station platform, he/she will detour to another walking path which few passengers are on.

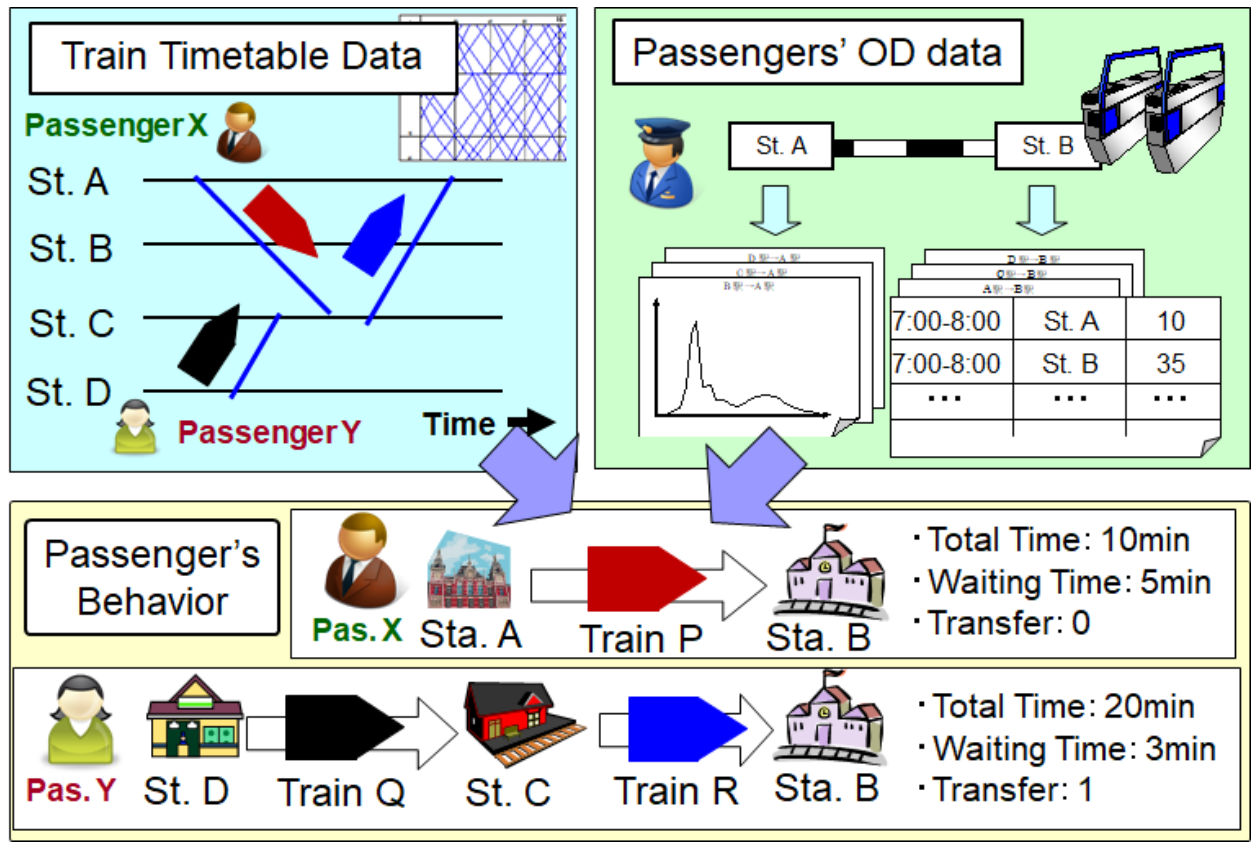

Figure 1: Structure of conventional train operation and passenger flow simulator.

\subsection{Purpose of the research}

We set the goal of the research as developing a new simulation system for train delay and passengers' comfort, satisfying the following requirements.

- Incorporating detailed passenger behaviour simulation about the situation of boarding and alighting, considering passengers' temporal alighting, and detour walking path for avoiding congested area.

- Estimating each train's delay and each passenger's total comfort under a timetable to evaluate the daily train operation plan within the practical calculation time

We focus on estimating train delay and passengers' comfort under a daily train traffic condition, without any disturbance caused by any external factors like rolling stock failure, humans/animals/vehicles presence on the line, or bad weather. They are rare cases in commuter lines in Japan. In addition, we are going to apply the new simulator for designing and scheduling a better train timetable in the planning stage. 


\subsection{Procedure of the research}

The procedure of the research is as follows. First, we conduct questionaries' survey for passengers' preference of position inside train car. Then, we develop detailed boarding and alighting simulation of passengers for a certain door of a train at a certain station. Finally, we combine the boarding and alighting simulation, passenger's choice behaviour for vehicles, and existing train operation and passenger flow simulator, to realize prediction for train delay and passengers' comfort under a certain timetable on a certain rail line.

\section{QUESTIONNAIRE SURVEY FOR PASSENGERS' POSITION INSIDE TRAIN CAR}

\subsection{Purpose of the survey}

In the questionnaire survey, we clarify passengers' preference for the position inside train car. As we described, the position where an alighting passenger is standing/sitting may influence the necessary time for alighting. If he/she is standing inner part of train car, it takes long time to get off, and some other passengers may temporarily alight the train car not to disturb him/her. As passengers' preference for the position inside train car seems to be different according to the congestion inside train car, we asked the respondents for preferences under several conditions.

\subsection{Procedure and questions of the survey}

The survey was conducted on the websites during 10 days in January 2018. We first conducted preliminary survey to select target respondents who almost always use commuter lines in Tokyo. On the main survey, 1,047 respondents sent the answers for our questions.

In the preliminary survey, we asked his/her time period, rail line, origin and destination stations, and existence of accompany persons in his/her daily use of railway to go to work from home. We picked up 1,748 respondents who use the commuter lines of inbound direction in Tokyo during 7 to $10 \mathrm{AM}$, and there are no accompany persons.

In the main survey, we first asked his/her standing/sitting position inside train car in the ordinal commuting condition. We also asked his/her riding time, congestion of train cars when boarding or alighting, his/her choice of vehicles, and the reason for the answers. Then, we asked his/her standing position under the imaginary conditions of congestion.

\subsection{Outline of the results}

In this paper, we describe about ordinally standing position inside train car. In the main survey, we set the alternatives of standing position inside train car (Fig. 2). Each respondent answered the alternative which he/she often stand. The results were shown in Fig. 3. The position most passengers chose was 1 . Following this, 3, 2, and 8 tended to be chosen by respondents. The result implies that, many passengers want to stand near the door to get off as fast as possible. It also suggests that the position close to the seats is preferable than that between the seats.

In addition, although this is not described in Fig. 3, when passengers' riding time become longer, position 2 become preferable than position 3. Also, when train congestion become greater, position 4 is preferable than position 7 or 8 . So, it can be said that passengers seem to decide their standing position considering not only easiness for get out, but also comfort and duration of standing in the congested train car. 


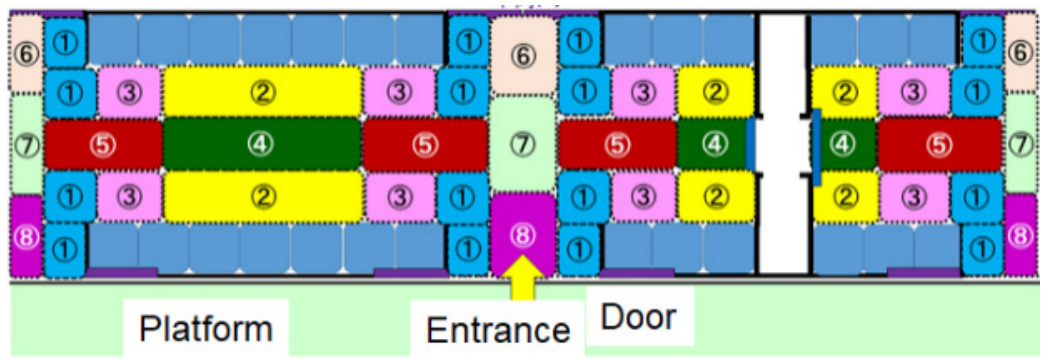

Figure 2: Alternatives for standing position inside train car.

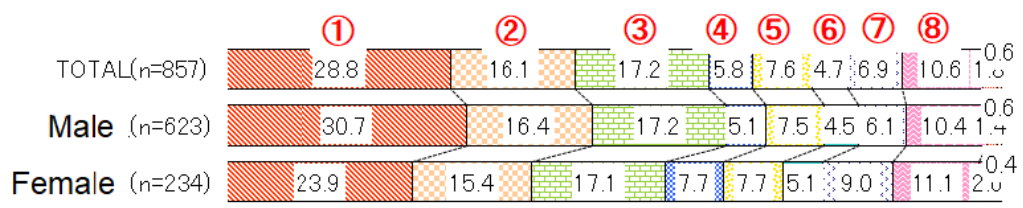

Figure 3: Rate of respondents about ordinally standing position inside train car.

\section{PASSENGER SIMULATION IN CASE OF BOARDING AND ALIGHTING}

\subsection{Outline of the simulation}

We developed detailed simulation of boarding and alighting for a certain door in a train at a station. The outline of the simulation is shown in Fig. 4. Passengers' movements for boarding and alighting are represented by transitions between the grids which are set by separating area both inside and outside of train car. Two or more passengers may be assigned on the same grid at the same time.

First, we set the number of passengers boarding, alighting and staying in train car for a certain door on the target situation. We can choose either setting the number of passengers directly, or using the number of passengers estimated by train operation and passenger flow simulator.

Next, initial position of each passenger in train car is decided one by one by using the adjusted score of passengers' preferences about standing/seating condition. The basic score of passengers' preferences is set reflecting the results of questionnaires described in section 3. The score is adjusted reflecting the number of other passengers on the surrounding grids.

For the third procedure, simulation for alighting is conducted until all alighting passengers get off from the train door. Passengers who temporarily get off are also decided in this step.

After that, passengers remaining in train car are relocated before boarding passengers come in. That is, after all alighting passengers get off, if there is more attractive grid for a passenger, he/she moves to the place before the boarding passengers come in.

Then, simulation for boarding is conducted by every second. Each boarding passenger decides his/her destination position by using adjusted score of preferences. When all passengers pass the train door, the boarding simulation ends.

The simulator outputs result of simulation, not only alighting and boarding time, but also all passengers' logs about grid transitions. By using passengers' logs, we can also estimate each passenger's comfort during boarding and alighting. 
4.2 Estimation of each passenger's walking path in case of boarding and alighting

In this section, we describe the detailed method for estimating each passenger's walking path in case of boarding and alighting. First, we describe the way for setting initial position in Fig. 5. Initial position of each passenger is set one by one in order of arrival. On the left-hand side of Fig. 5, the basic preference score and position of other passengers who are already disposed are illustrated. The basic preference score for a certain grid is adjusted reflecting the number of passengers surrounding it. The adjusted score is shown on the right-hand side. The next passenger is disposed on the grid with the highest adjusted score.

Then, we describe the method for searching a walking path of alighting passenger towards the door. We conduct shortest path search. The structure of grids is converted into indirectional graph network as described in Fig. 6. The costs of edges are set reflecting the number of other passengers on the neighbouring grids. By estimating the shortest path, passenger's detour behaviour not to pass congested area is reproduced. In case if there is a grid on which more than X shortest paths of alighting passengers pass, the staying passengers on that grid become temporal alighting, because they disturb alighting passengers. In addition, as the shortest paths are changed along with changes of passengers' positions at a certain time, they are recalculated at every simulation clock, by using fast estimation algorithm for the shortest paths [5].

In the simulation for alighting, each passenger proceeds basically a grid per second on his/her shortest path. In case when two or more passengers are located on the same grid at the same time, they have "one-time rest", and do not proceed on the grid in the next time step. In this way, we can represent the situation that it takes time for passengers to pass congested area.

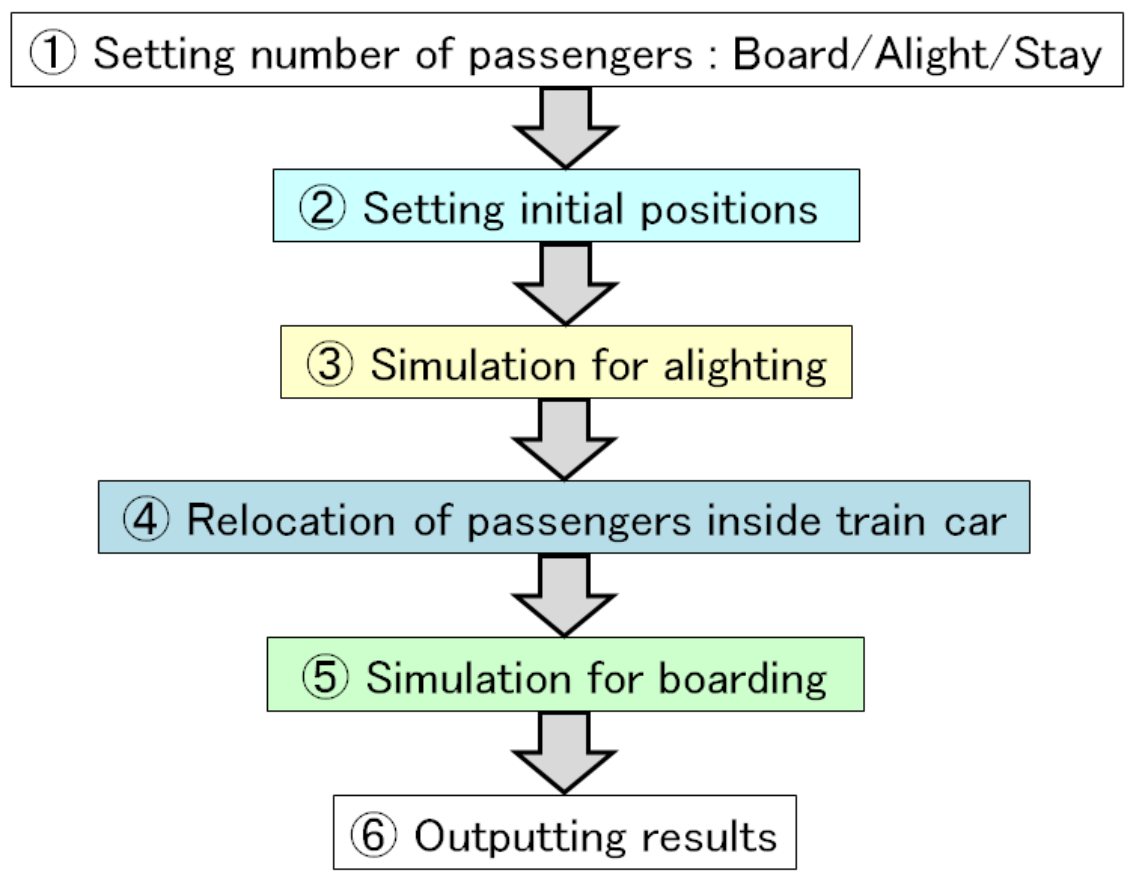

Figure 4: Outline of passenger simulation in case of boarding and alighting. 


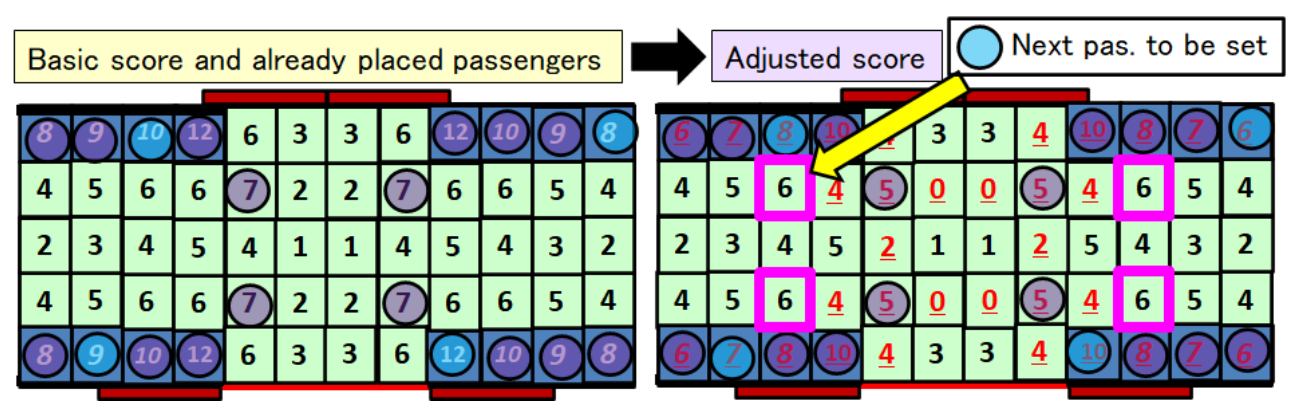

Figure 5: How to set initial position of passengers (Step 2).

Pas. for temporal alighting are decided when they disturb alighting Pas.

Alighting Pas. pass non-congested area to alight as fast as possible

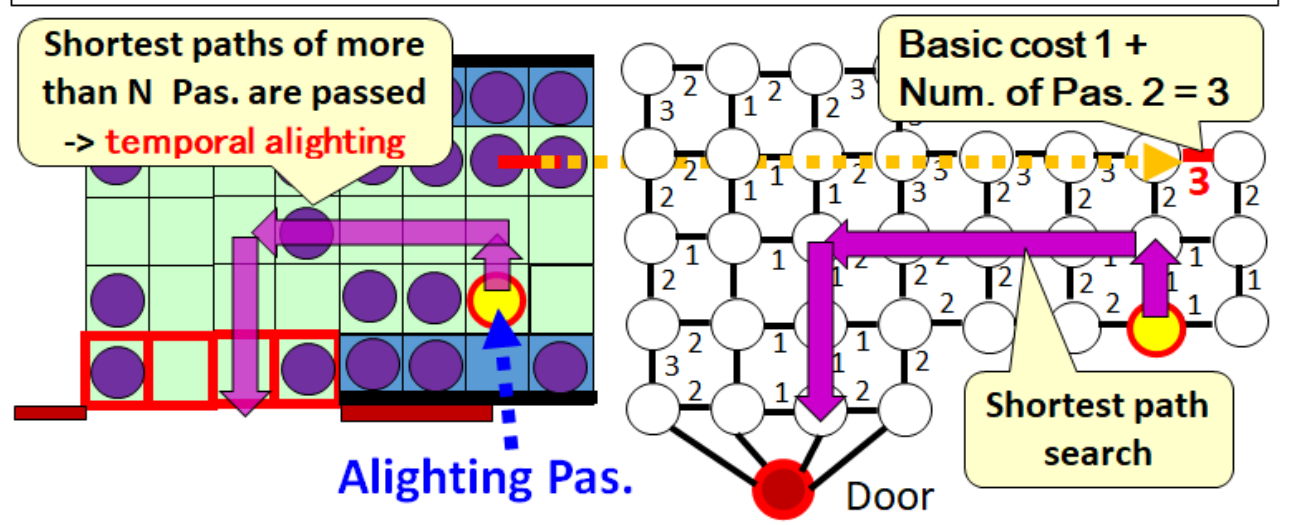

Figure 6: Simulation for alighting (Step 3).

In step 4, passengers remaining in train car are relocated. This is done based on the adjusted preference score for each passenger. Fig. 7 shows an example of adjustment. After all alighting passengers get out, adjusted preference score for each passenger is calculated. It reflects not only basic score and the number of passengers on neighbouring grids, but also the distance from the grid which he/she is now on. It represents the situation that, although a staying passenger in train car want to move more preferable position, as he/she do not want to walk long distance, he/she change his/her position only if more preferable position is occurred near his/her current position.

Fig. 8 illustrates estimation of walking paths for the boarding passengers. The target position inside train car for each boarding passenger is decided along with the adjusted preference score. Then, the shortest path search is conducted by the same way as that for the alighting passengers. 


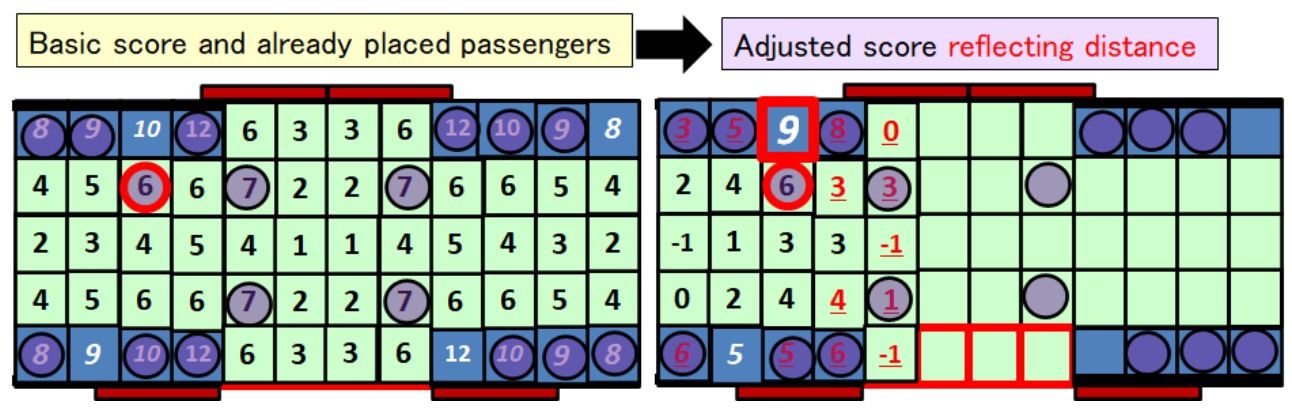

Figure 7: Adjusting preferences of position according to the number of passengers (Step 4).
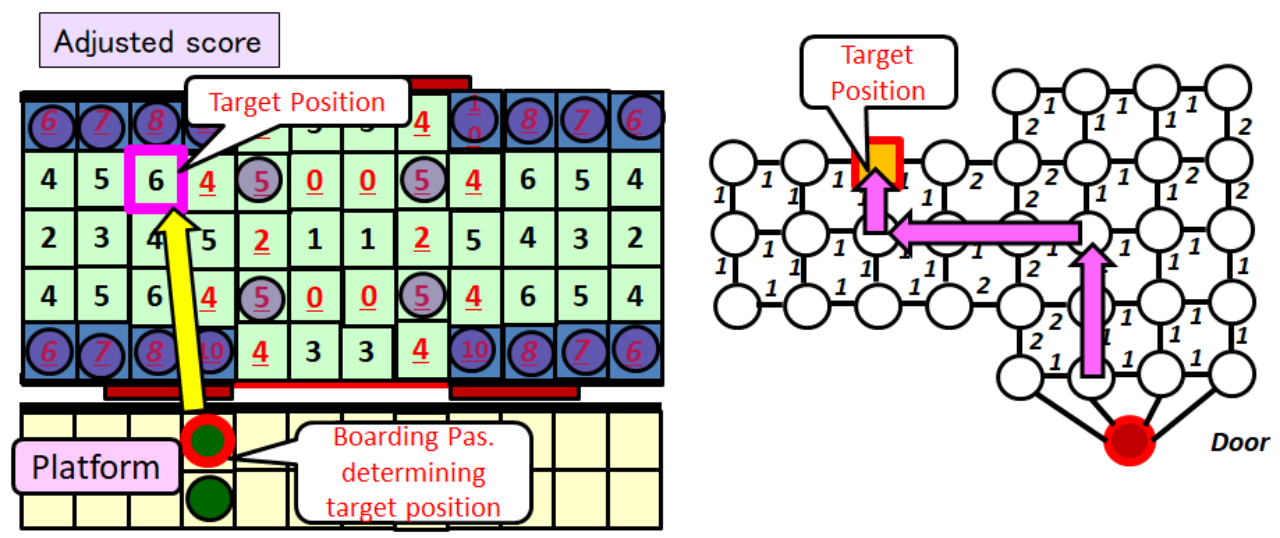

Figure 8: Simulation for boarding (Step 6).

We can consider the influence of some passengers standing near the doors. When we decide a walking path of an alighting or boarding passenger, the shortest path makes a detour for them. In case when there are no detour way, he/she has to walk near them, but when his/her and they are on the same grid, he/she takes "one-time rest", which results additional time for alighting or boarding. As a consequence, the necessary time for boarding and alighting become longer, compared with the situation without such standing passengers near the door.

On the other hand, there are other internal factors affecting boarding and alighting time. For example, the number and size of passenger luggage and their allocation inside the train car may influence on the dwell time, in case of high-speed or inter-city trains. Also, the presence of passengers waiting for the next train on the same platform may also influence on the dwell time. In this research, our target situation is peak hours in commuter rail lines, like metro inside the city. There are no such inter-city trains. Only few passengers have a big luggage. The number of passengers waiting for the next train on the same platform is also limited. So, in this research, we do not consider such influences. 


\subsection{Case study of boarding and alighting simulation}

We will show some an example of boarding and alighting simulation. Fig. 9 illustrates the case that the number of alighting passengers is 30 , that of boarding 30 , that of staying 30 . It firstly determines temporal alighting passengers by using the results of shortest path searches for the alighting passengers. In this case, we suppose that if more than three alighting passengers pass a certain grid, staying passengers on that grid become temporal alighting. For the result of this case, 4 passengers become temporal alighting.

The left-hand side of Fig. 9 illustrate the situation that all alighting passengers start to move for alighting just after the door open. On the right-hand side of Fig. 9 illustrate the situation that all alighting passengers start to move 10 seconds before the door open. Passengers' distributions when the door open are illustrated at the top of figure, and those 18 seconds after the door open are at the bottom. On the left-hand side, the alighting passengers are distributed when the door open. It affects necessary time for alighting, and there are still alighting passengers in case of 18 seconds after the door open. But, on the right-hand side, alighting passengers are concentrated when the door open. This contributes to shorten the necessary time for alighting. In case of 18 seconds after, all alighting passengers have already got off, and the boarding passengers have already started to get on. The total alighting and boarding time were 31 seconds, which is 6 seconds shorter than that in the left-hand side. So, we can conclude that it is effective for shorten dwell time to encourage passengers to start to move several seconds before the door open. In this way, we can quantitatively evaluate effects of countermeasures for decreasing train delay.

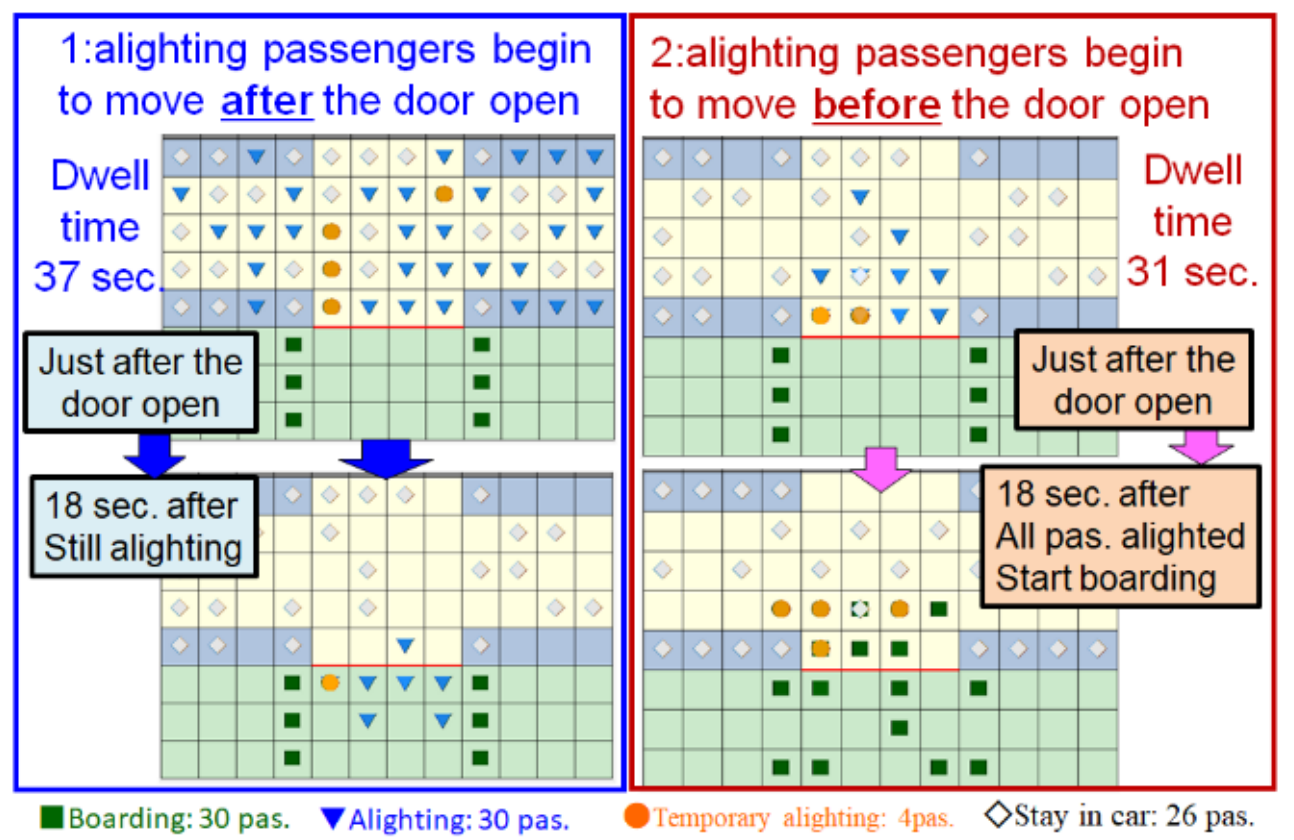

Figure 9: Comparison of boarding and alighting simulations. 


\subsection{Comparison of dwell time for boarding and alighting}

To validate the simulation from the viewpoints of necessary time for boarding and alighting, we compared estimated necessary time with actual data. There were data of necessary time for boarding and alighting in 35 experimental cases. These experiments were conducted in a mock-up train and platform. When we conducted the simulation, we set the same number of boarding, alighting and staying passengers as those in actual data. Because we did not know detailed condition in train car in the experiments, the simulation was conducted tens of times for a set of the number of passengers, varying initial position of alighting passengers probabilistically. We also changed the time when alighting passengers start to move to get off. For a comparison, we conducted regression analysis for actual 35 data, and prepare a regression formula. We calculated the dwell time in case of the conventional method by using the regression formula and the number of passengers in the actual data.

Fig. 10 illustrates the results. The horizontal axis indicates sum of the number of passengers getting on and off. The vertical axis indicates boarding and alighting time. The actual data about boarding and alighting time are indicated by " $x$ ". The estimated time by conventional and proposed method are indicated as " $\square$ " or "•". For the proposed method, boarding and alighting time are illustrated as interval estimation, because we conducted simulation tens of times varying conditions. The trend of estimation for the three cases are almost the same. By taking rate of cases within 10 seconds error, compared with actual ones, the proposed method become $94 \%$. That for the conventional method was $74 \%$. So, we can conclude that the proposed method has at least the same accuracy with the conventional method.

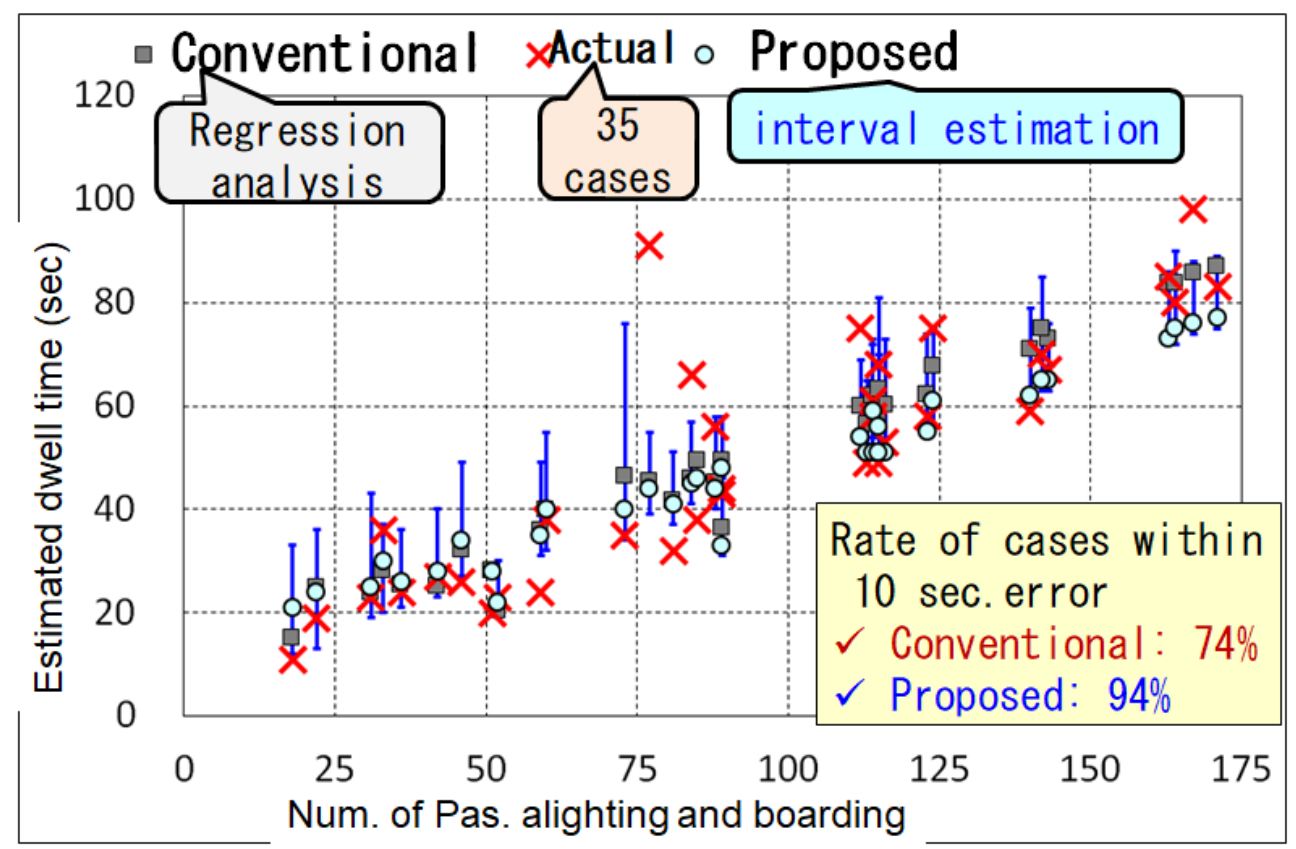

Figure 10: Comparison of estimated dwell time with actual ones. 


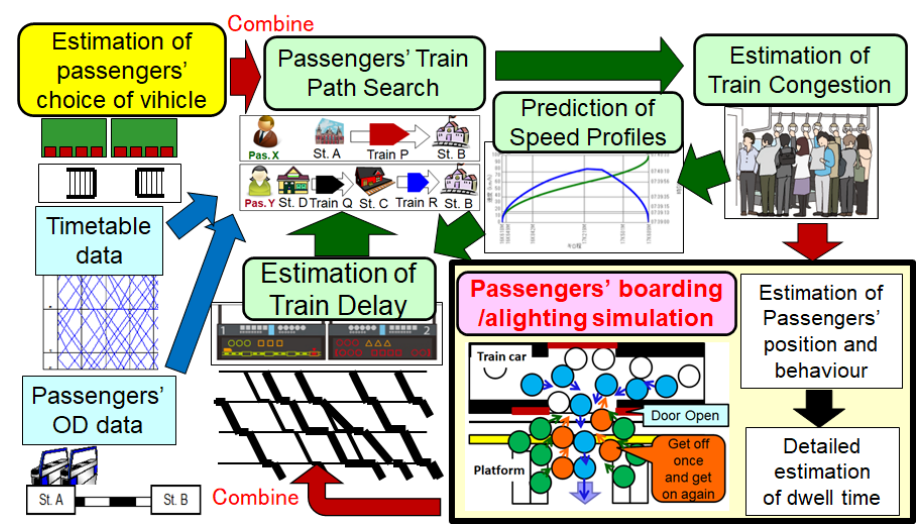

Figure 11: Overall structure of train operation and passenger flow simulator with linkage.

\section{LINKAGE WITH TRAIN OPERATION AND PASSENGER FLOW SIMULATOR}

To improve accuracy of estimation of train delay in train operation and passenger flow simulator, we combined the boarding and alighting simulation with the simulator. We also combined estimation function for passengers' choice of vehicles. It is decided by not only position of stairs in origin or destination station, but also the number of passengers lining up for the next train [6]. So, our passengers' choice model for vehicles is developed reflecting both structure and congestion of platform in each passenger's origin and destination station.

Fig. 11 shows the new structure with linkage. As train operation and passenger flow simulator can estimate each passenger's train path, reflecting his/her train choice for successive train and left behind, we can estimate dwell time by using more realistic number of boarding and alighting passengers. We can also estimate effects of reduction of train delay directly and quantitatively in a certain rail line by introducing some counter measures for smooth boarding and alighting. Especially in Japan, widening train doors, changing layout inside train car, or changing the way of lining up at station platform are often discussed and enforced to decrease train delay. By using the new simulator, railway companies can know how much delay can be decreased by a certain countermeasure in advance.

In addition, we can also evaluate the effects from the viewpoints of passengers. We can know how many times and how long a certain passenger stands close to other passengers. By estimating them, we can evaluate passengers' comfort quantitatively.

On the other hand, as we use passengers' data collected by automatic ticket gates at stations, some passengers may stop their itinerary between the ticket gates (e.g. for a coffee). In this research, as our target situation is peak hours in commuter rail lines, and few passengers will stop their itinerary during their commuting, we do not consider such break of itinerary.

\section{APPLICATION FOR EXISTING RAIL LINE}

\subsection{Outline of the rail line and preconditions}

We applied the updated train operation and passenger flow simulator for an actual commuter line in Japan. The rail line has 19 stations. The number of trains in the whole day is about 1,000 . In the peak hours, trains are set about every 4 minutes for one direction. 
We simulated and compared two countermeasures for train operation. One is widening doors of the train car. We assumed that width of all doors become $1.8 \mathrm{~m}$, which enables four passengers to get on or off at once. The other is a kind of passengers' manner for boarding and alighting. We assumed that all passengers become polite, and behave cooperative for other passengers' boarding and alighting. That is, when passengers get on a train, they willingly go to inner area of train car, and when they disturb other alighting passengers, they willingly alight temporarily. In addition to these conditions, we simulated train traffic and passenger flow under the current situation.

We calculated two evaluation indexes. One is maximum value of train delay. This is not for a certain train or a certain station, but for all trains and stations in the rail line. The other index is average neighbouring time with other passengers. This is based on the situation in which more than two passengers are on the same grid in the boarding and alighting simulation.

The time during which a certain passenger is involved in such situation is calculated and summed up. Then, we take an average for all passengers in the rail line. The latter index indicates each passenger's comfort during boarding and alighting. By summing up the time for all his/her journey, we can estimate his/her comfort during the trip. We can evaluate the total level of comfort for a rail line by taking average for all passengers.

\subsection{Results of test calculations}

The results of test calculations are summarized in Table 1 . The simulation was conducted from the earliest train in the morning to noon. The maximum values of train delay were calculated by taking all trains running before $10 \mathrm{AM}$. The average neighbouring time with other passengers are calculated for about 280,000 passengers using the line before $10 \mathrm{AM}$.

From the viewpoint of punctuality, maximum train delay was estimated to drop by 25 seconds with widening train doors. The effect was estimated to be 24 seconds in case of passengers' cooperative behaviour. But, in the latter case, the condition that all passengers take cooperative behaviour is supposed. So, we have to understand the results that the effects are at most 24 seconds. If we want to realize further decrease of train delay, we have to discuss other countermeasures like widening doors.

On the other hand, from the viewpoints of passengers' comfort, average neighbouring time will drop significantly when widening train doors. When passengers take cooperative behaviour, average neighbouring time is slightly dropped. So, it can be said that, by taking cooperative behaviour, passengers can enjoy better transportation services from the viewpoints of not only punctuality, but also comfort. These results will encourage passengers to take cooperative behaviour during boarding and alighting.

Table 1: Comparison of the simulated results.

\begin{tabular}{|l|c|c|}
\hline & $\begin{array}{c}\text { Maximum train } \\
\text { delay (seconds) }\end{array}$ & $\begin{array}{c}\text { Average neighbouring } \\
\text { time with other } \\
\text { passengers (seconds) }\end{array}$ \\
\hline 1. Current condition & 102 & 25.9 \\
\hline 2. Widen train doors & 77 & 21.7 \\
\hline 3. Passengers' cooperative behaviour & 78 & 24.3 \\
\hline
\end{tabular}




\section{CONCLUSION AND FUTURE WORKS}

In this research, to estimate effects of reduction of train delay by taking countermeasures for smooth boarding and alighting, we have developed simulation for boarding and alighting. It can consider detailed situation of boarding and alighting, like temporal alighting not to disturb other alighting passengers. It also reflects passengers' preferences of standing position based on the results of questionaries' survey. Then, it combines with existing train operation and passenger flow simulator, which enable us to estimate changes of train delay or passenger comfort quantitatively. By the test calculation, the effects are estimated in an actual commuter rail line.

For the future works, we will apply the simulator for many other rail lines, and validate the accuracy or usefulness of that. Especially, in rail lines including long-distance transportation like high-speed or inter-city, we have to extend the simulation model to incorporate passenger luggage, and existence of passengers waiting for the successive train on the same platform. They are not so difficult to incorporate such internal factors, because existence of luggage or waiting passengers can easily be added on the boarding and alighting simulation. We also consider passenger's temporal stop of his/her journey. It will become a considerable work, because we first have to clarify passengers' trend for stopping their itinerary. In addition, we are going to model other layouts in train car, like cross seats, and compare the results along with the difference of layouts. By incorporating these further internal factors affecting dwell time, the simulation can become realistic, and be applied for more complicated situation. Moreover, from the viewpoints of further external factors, we will extend the simulation for disrupted condition, like rolling stock failure or bad weather, to evaluate train rescheduling.

\section{REFERENCES}

[1] Tomii, N., Techniques To Make Train Diagrams, Ohmsha Publishing, 2012. (In Japanese).

[2] Kunimatsu, T., Hirai, C. \& Tomii, N., Evaluation of timetables by estimating passengers' personal disutility using micro-simulation. Proceedings of the $3 \mathrm{rd}$ International Seminar on Railway Operations Modelling and Analysis: RailZurich2009, 2009.

[3] Oto et al., A study on passenger flow in a railway station (in Japanese). Summaries of technical papers of Annual Meeting Architectural Institute of Japan, E-1, pp. 845-846, 1999.

[4] Iwakura, S. et al., A multi agent simulation model for estimating knock-on train delays under high-frequency urban rail operation. Transport Policy Studies' Review, 15(4), 2013. (In Japanese).

[5] Tatsui, D., Kunimatsu, T. \& Sakaguchi, T., Interactive timetabling system with the function of estimating train congestion on timetable planned. Proceedings of the 14th International conference on Railway Engineering Design and Optimization (COMPRAIL2016), 2016.

[6] Fang, J. et al., A model of passenger distribution on metro platforms based on passengers' boarding strategies. Proceedings of 8th International Conference on Railway Operation, Modelling and Analysis: RailNorrköping2019, 2019. 